\title{
Spectral Clustering as a Diagnostic Tool in Cross-Sectional MR Studies: An Application to Mild Dementia ${ }^{\star}$
}

\author{
Paul Aljabar ${ }^{1}$, Daniel Rueckert ${ }^{1}$, and William R. Crum ${ }^{2}$ \\ ${ }^{1}$ Department of Computing, Imperial College London, UK \\ ${ }^{2}$ Centre for NeuroImaging Sciences, Institute of Psychiatry, \\ Kings College London, UK
}

\begin{abstract}
Structural imaging investigations commonly apply a segmentation step followed by the extraction of feature data that can be used to compare or discriminate groups. We present a framework for such a study based on automated multi-atlas segmentation followed by the extraction of low-level morphological features, volumes and overlaps, for classification. A spectral analysis step is used to transform pairwise overlap information into feature data that relate to individual subjects. Applying the framework to a group of controls and patients with mild dementia, we compare the volume- and overlap-based classification performance using both supervised and unsupervised classifiers. The results indicate that unsupervised classification following a spectral analysis of label overlaps performs very well, outperforming classifiers that use volumes alone.
\end{abstract}

\section{Introduction}

Structural MR imaging is often used to derive morphological markers which discriminate subjects or groups on the basis of information relating to pathology, cognition, genetics etc. In the context of dementia, significant progress has been made in recent years, in parallel with advances in image acquisition and in image analysis. Structural studies of dementia can utilise simple volumetry [1] or more sophisticated shape-based measures of morphology [2]. Segmentations are usually required and manual segmentations continue to be regarded as the gold standard, especially for challenging structures such as the hippocampus (see [1] for example). There has, however, been a steady improvement in the accuracy and reliability of automated segmentation techniques (see 3 for example) making them an increasingly viable alternative to time-consuming manual methods. While longitudinal studies are likely to remain the most powerful way of investigating dementia, there has been a steady improvement in cross-sectional neuromorphological studies as associations are identified between particular brain structures and the onset and progress of dementia (see e.g. [4|5]6]).

\footnotetext{
* This work was carried out with the support of the EPSRC (GR/S82503/01 and GR/N14248/01) and the MRC (D2025/31). We are grateful to Nick Fox (Dementia Research Centre, Institute of Neurology, London, UK) and to David Kennedy (Centre for Morphometric Analysis, MGH, MA) for providing the study and training data.
} 
In this work, we use features derived from low level morphological descriptors to analyse a group of AD patients and matched controls. The study applies automated segmentation to image data acquired at a single timepoint. We use two types of features: (a) subject-specific morphological quantities and (b) measures of morphological similarity between subjects. The simplest possible subject-specific feature descriptors are based on the volumes of anatomical structures. We also use morphological features to measure the pairwise similarity of structures employing the concepts of simple and generalised overlaps [7] which characterise the pairwise similarity of single or multiple structures across subjects. Techniques from spectral analysis are used to convert overlaps from measures of pairwise similarity between subjects into per-subject feature data for classification. Additionally, we compare supervised and unsupervised classification methods using the feature data and make an assessment of classification rates for different combinations of feature data type and classifier, comparing with classification rates published in the literature.

\section{Data and Methods}

The framework presented is applied to a set of study images that are assigned to clinical groups consisting of patients diagnosed with Alzheimer's disease (AD) and matched controls. The images are first segmented to create labeled structures which are then used to extract feature data for a classification step. An overview of the analysis pipeline is shown in Figure 1.

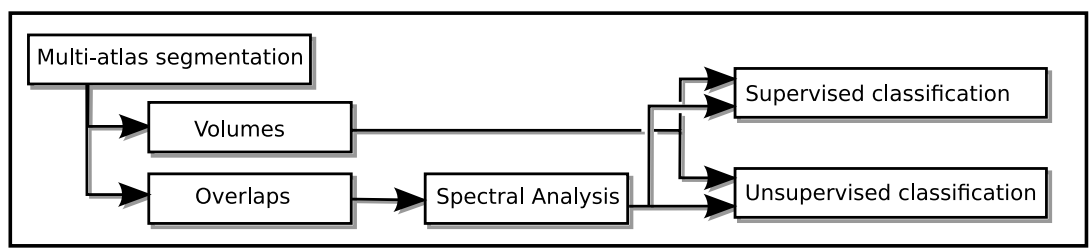

Fig. 1. A schematic illustration of the different components of the analysis pipeline

\subsection{Multi-atlas Segmentation}

Automated segmentation of the study group images is achieved by the propagation and fusion of atlases from a separate database consisting of 275 T1-weighted MR brain images of subjects aged between 4 and 83 years. Multiple centres provided the images which were manually segmented into 17 subcortical structures at the Centre for Morphometric Analysis (MGH, Charlestown, MA). The structures segmented were: Lateral ventricle, thalamus, caudate, putamen, pallidum, hippocampus, amygdala, accumbens, hippocampus, brainstem. 

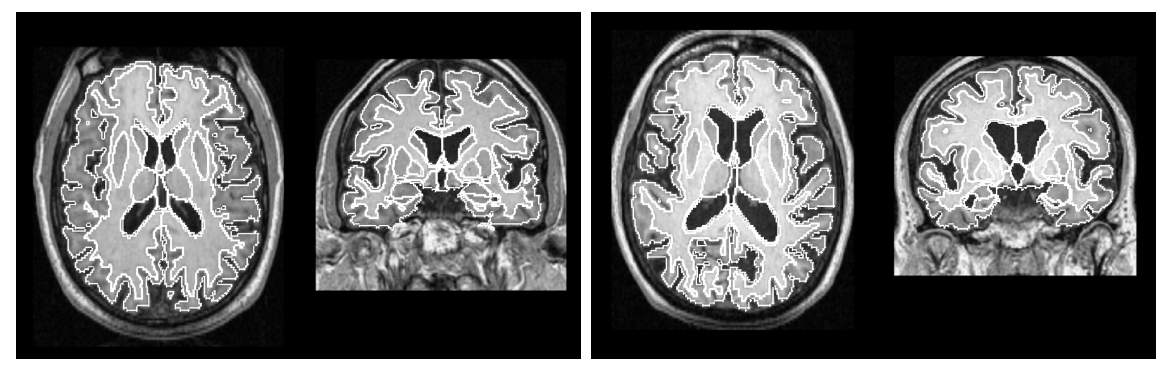

Fig. 2. Segmentation examples for a control subject (L) and a patient (R)

Non-rigidly propagating and fusing atlas labels has been shown to accurately segment human brain structures [3]. Additionally, due to the large number of database images and the desirability of using atlases appropriate for each study image, a subset of atlases were selected from the entire database of 275 atlases for each subject in the study group. Atlases were ranked by their normalised mutual information with the target and the 20 top-ranked atlases were propagated and fused using majority voting at each voxel. This selection approach to multi-atlas segmentation has previously been shown to be effective 8. Some subjects in the study group differed from the atlas database in having significantly larger internal cerebro-spinal fluid (CSF) spaces. Therefore a correction was applied to each image using an expectation-maximisation based tissue classification [9] to relabel as CSF any voxels previously labeled as tissue but with a high CSF membership probability $(\geq 0.75)$. Example segmentations for a patient and a control subject are shown in figure 2 .

\section{$2.2 \quad$ Feature Extraction}

Segmentations for each subject allow features to be extracted for classification. The volumes of the segmented structures represent the simplest level of morphological description and overlaps measuring agreement between subjects for a structure represent an incrementally higher level of description.

After spatial normalisation, the volumes of each structure across the subjects were found and converted to z-scores to accommodate differences in scale between structures. For $K$ structures, the $N \times 1$ vector $\mathbf{v}_{i}$ contains the volume Z-scores for structure $i, 1 \leq i \leq K$ and these vectors can be used to create the matrix $\left[\mathbf{v}_{1} \ldots \mathbf{v}_{K}\right]$ with rows that provide vector feature data for the group. Overlaps were calculated using the Dice coefficient $d=\frac{2|A \cap B|}{|A|+|B|}$ where $A$ and $B$ represent the regions of a particular labeled structure within a pair of images after spatial normalisation to correct for gross differences. Agreement between two subjects across a group of structures can be measured with generalised overlap (Crum et al. 7). This was used to aggregate overlaps for multiple structures with weights inversely proportional to their volume. 


\subsection{Spectral Analysis}

The structure overlaps for each pair of spatially normalised segmentations, which represent pairwise similarities between subjects, must be converted into persubject feature data before use for classification. This is possible with a spectral analysis step in which the subjects are viewed as the nodes of a complete weighted graph where each edge's weight is defined by the similarity of the subject pair it joins. A good introduction to spectral analysis is given by von Luxburg [10. The graph for $N$ subjects (nodes) defines the symmetric weights matrix $\mathbf{W}=\left(w_{i j}\right)$, $i, j=1, \ldots, N$ where $w_{i j}$ is the similarity of subjects $i$ and $j\left(w_{i i}=0\right)$. The diagonal degree matrix $\mathbf{D}$ is given by $D_{i i}=\sum_{j=1}^{N} w_{i j}$ and the $N \times N$ normalised Laplacian matrix $\mathbf{L}$ is given by $\mathbf{L}=\mathbf{D}^{-\frac{1}{2}}(\mathbf{D}-\mathbf{W}) \mathbf{D}^{-\frac{1}{2}}$. It can be shown that ordered eigenvalues $\lambda_{1}, \ldots, \lambda_{N}$ of $\mathbf{L}$ satisfy $0=\lambda_{1}<\lambda_{2} \leq \lambda_{3} \leq \ldots \leq \lambda_{N}$. Let the corresponding eigenvectors be denoted $\mathbf{w}_{1}, \ldots, \mathbf{w}_{N}$. It can be shown that $\mathbf{w}_{1}$ has equal components and it is not used for classification. The remaining $\mathbf{w}_{i}$ can, however, be used to generate per-subject feature data. Define the $N \times m$ feature matrix $\mathbf{F}_{m}(\mathbf{L}), m<N$, by taking the first $m$ eigenvectors from $\mathbf{w}_{2}, \ldots, \mathbf{w}_{N}$ as columns and normalising the rows to one. The rows of $\mathbf{F}_{m}(\mathbf{L})$ provide a feature vector for each subject. If $m=1, \mathbf{F}_{m}$ only uses information from the first available eigenvector for classification, $\mathbf{w}_{2}$, which is termed the 'Fiedler' vector. If $K$ structures are available, we construct separate Laplacian matrices $\mathbf{L}_{1}, \ldots, \mathbf{L}_{K}$ for each structure and combine the feature data from separate Laplacian matrices to create the $N \times m K$ feature matrix $\left[\mathbf{F}_{m}\left(\mathbf{L}_{1}\right) \ldots \mathbf{F}_{m}\left(\mathbf{L}_{K}\right)\right]$. Instead of combining feature data from separate Laplacian matrices, it is possible to first aggregate Dice coefficients for a group of structures into a generalised overlap for each subject pair. These provide a single Laplacian matrix $\mathbf{L}_{g e n}$ whose eigenvectors provide $m$-dimensional feature data in the form of $\mathbf{F}_{m}\left(\mathbf{L}_{g e n}\right)$. The more compact notation $\mathbf{F}_{m, i}$ and $\mathbf{F}_{m, g e n}$ will be used below for $\mathbf{F}_{m}\left(\mathbf{L}_{i}\right)$ and $\mathbf{F}_{m}\left(\mathbf{L}_{g e n}\right)$.

\subsection{Supervised and Unsupervised Classification}

The different forms of feature data were used in conjunction with two types of classifier, one supervised and one unsupervised, for the final step in the analysis. These were the Fisher Linear Discriminant (FLD) and Fuzzy c-means (details may be found in 11], for example). Supervised FLD classification seeks a direction in the data that best separates the groups when clinical status is known. Unsupervised c-means clustering seeks a prescribed number of clusters (2 for our data) without using the clinical status and such that the within cluster variance is minimal. Each data point has fractional cluster membership which is thresholded on convergence to give a hard assignment. FLD classification rates were obtained using a leave-one-out procedure while c-means was applied to all the feature data and the resulting clusters were compared with the clinical groups. The conversion of volumes to z-scores ensures that the c-means clustering is not dominated by larger structures with greater variation. 


\section{Data and Experiments}

The study data comprises 38 patients with early AD symptoms and 19 matched controls. The Mini-Mental State Exam scores for the patients is in the range $13-26$ and $\geq 27$ for controls. Group ages are $69.8 \pm 7$ for patients and $69.3 \pm 7$ for controls. There are 23 (10) women in the patient (control) group. Further detail of the study data is given in 12. Each image in the study group is segmented as described in Section 2.1 and the segmentations are spatially normalised to the MNI single subject template before evaluating volumes and overlaps. This is carried out using affine and low dimensional non-rigid registrations modeled using free-form deformations with a control point spacing of 20mm [13]. Volumes of all structures for each subject and overlaps for each structure across all subject pairs are then calculated and used to generate feature data as described in sections 2.2 and 2.3 . Experiments are then performed to assess classification performance using the volume- and overlap-derived features.

With seventeen sub-cortical labels, there are a large number of subsets for generating feature data and an exhaustive analysis using all subsets is not feasible within this submission. Moreover, given that particular regions have been associated with the progress of AD (see e.g. 445]), a restricted subset of the structures is used based on their individual ability to discriminate the clinical groups. Unpaired two sample t-tests are used to measure AD/control discrimination for each structure and the five structures with the highest absolute t-values are defined as the most discriminating. This is carried out separately using the components of each volume vector $\mathbf{v}_{i}$ and each overlap-derived feature $\mathbf{F}_{1, i}$ for $1 \leq i \leq 17$.

The first experiment used the chosen structure subsets to generate feature data matrices $\left[\mathbf{v}_{1} \ldots \mathbf{v}_{5}\right]$ and $\left[\mathbf{F}_{1,1} \ldots \mathbf{F}_{1,5}\right]$ which allows a comparison of classification performance between volumes and overlaps based on feature vectors with equal dimension. Classification was also carried out with feature data using $m$ eigenvectors from separate Laplacian matrices, $\left[\mathbf{F}_{m, 1} \ldots \mathbf{F}_{m, 5}\right]$, for different choices of $m$. A comparison was made between the rates obtained by Fiedler vectors derived from separate Laplacians, $\left[\mathbf{F}_{1,1} \ldots \mathbf{F}_{1,5}\right]$, and the Fiedler-based features $\mathbf{F}_{1, \text { gen }}$ derived from the generalised overlap Laplacian matrix $\mathbf{L}_{\text {gen }}$. Finally, classification was assessed when using multiple eigenvectors from $\mathbf{L}_{g e n}$ to give the $m$ dimensional feature data $\mathbf{F}_{m, g e n}$ for $m>1$.

\section{Results}

\subsection{Structure Selection}

Based on absolute t-statistics, the five most discriminating structures using volume data $\mathbf{v}_{i}$ are: left thalamus $(t=4.1)$, right hippocampus, right thalamus, left hippocampus and right pallidum $(t=3.2)$. The most discriminating structures using $\mathbf{F}_{1, i}$ are: left hippocampus $(t=5.3)$, right hippocampus, right lateral ventricle, left thalamus and right thalamus $(t=3.3)$. These structures define the subsets used for the results below for volume- and overlap-derived feature data. 
All subjects are used for t-statistic calculation although the sets of most discriminating structures changed very little when the t-statistics were calculated leaving out each of the 58 subjects in turn.

\subsection{Classification}

Classification performance based on the volumes $\left[\mathbf{v}_{1} \ldots \mathbf{v}_{5}\right]$ and the overlapderived feature data $\left[\mathbf{F}_{1,1} \ldots \mathbf{F}_{1,5}\right]$ is summarised in Table 1$]$ which shows that overlaps outperform volumes for classification. This is consistent with the view that overlaps provide a higher level of morphological description. The feature data $\left[\mathbf{F}_{1,1} \ldots \mathbf{F}_{1,5}\right]$ give equal performance whether the classifier is supervised or unsupervised.

Table 1. Classifier performance figures based on the use of feature data derived from volumes $(\mathrm{V})$ and overlaps $(\mathrm{O})$ with a supervised and an unsupervised classifier

\begin{tabular}{|l|c|c|c|c|c|c|}
\hline & \multicolumn{2}{|c|}{ Specificity (\%) } & \multicolumn{2}{c|}{ Sensitivity (\%) } & \multicolumn{2}{c|}{ Rate (\%) } \\
\hline Classifier & $\mathrm{V}$ & $\mathrm{O}$ & $\mathrm{V}$ & $\mathrm{O}$ & $\mathrm{V}$ & $\mathrm{O}$ \\
\hline supervised & 79 & 89 & 74 & 82 & 76 & 84 \\
\hline unsupervised & 79 & 89 & 77 & 82 & 78 & 84 \\
\hline
\end{tabular}

The use of FLD classification allows a visualisation of the group separation with respect to the five-dimensional feature datasets based on volumes and overlaps. The FLD classification for each dataset provides a direction of maximum separation onto which the feature data can be projected. Box-plots showing the projected volume- and overlap-based data are shown for each group in Figure 3 .

Classification rates from overlaps did not generally improve by using multiple eigenvectors from each of the structures' separate Laplacian matrices, i.e. $\left[\mathbf{F}_{m, 1} \ldots \mathbf{F}_{m, 5}\right]$ for $m>1$. Only one example gave a higher overall rate but this
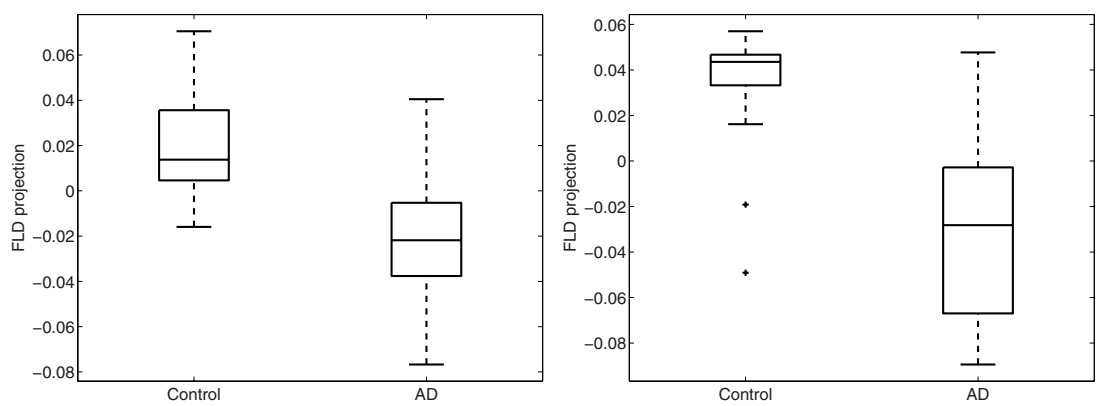

Fig. 3. Visualising group separation using FLD projections of the feature data. Left: Volumes as feature data. Right: Overlap-derived feature data using spectral analysis. 
was at the expense of a significant drop in sensitivity ( $m=6$, c-means, rate: $86 \%$, specificity: $92 \%$, sensitivity: $73 \%$ ).

The best rates using combined features from separate Laplacian matrices were obtained using single Fiedler vectors $\left(\left[\mathbf{F}_{1,1} \ldots \mathbf{F}_{1,5}\right]\right)$. Therefore the natural comparison was made with the use of a Fiedler vector from a combined overlap Laplacian matrix $\mathbf{L}_{\text {gen }}$. The classification rate using the feature $\mathbf{F}_{1, \text { gen }}$ was, however, disappointing (76\%, supervised and unsupervised). This result is, perhaps, unsurprising given that classification using five-dimensional feature vectors was compared against classification using a scalar feature. Generalised overlap classification performance improves significantly, however, by using the higher dimensional data $\mathbf{F}_{m, \text { gen }}$ where $m>1$. The best performance was obtained for $m=8$ where the sensitivity, specificity and overall classification rate were $89 \%, 92 \%$ and $92 \%$ respectively using c-means. The corresponding values using FLD were $89 \%, 84 \%$ and $86 \%$. These figures show that rates improve for both supervised and unsupervised classifiers when vector feature data based on generalised overlaps are used.

\section{Discussion}

We have presented a framework in which low-level features are used for clinical classification within a cross-sectional (single timepoint) study. In particular we describe a novel application of spectral analysis to structural segmentations which discriminates very well between patients with mild dementia due to AD and matched controls. The highest classification rates are obtained by unsupervised clustering of features based on the generalised overlap of a selection of structures. These were comparable to rates obtained using state-of-the-art methods such as those reported by Klöppel et al. 5. who exploit recent advances in statistical learning theory. It is worth noting that, in contrast with the data in [5, our AD subjects scored slightly higher on MMSE, were not confirmed post-mortem and were, on average, ten years younger.

The inter-subject similarity measures used for spectral analysis in this work are simple and generalised overlaps of structures estimated by multi-atlas segmentation. We have also used inter-subject differences in structure volumes to define the similarity and the feature data they generate give classification rates broadly between those given by volumes and overlaps (the volume difference results are not reported for reasons of space). Future work may investigate other possible forms for the similarity which can be derived from local segmentation information or features of the image data.

Overlap feature data were shown to better distinguish the groups than those derived from volumes. Reasons for this may include the higher level of description offered by structural overlaps and the fact that spectral analysis makes use of all pairwise similarity relations among the subjects. A few variants of spectral analysis exist with little current guidance on their optimal application. Our best results were obtained using the Normalised Laplacian as described in [10]. 
Applying the presented framework to new data and/or similarity metrics may require steps to identify the best form of spectral analysis to use.

Unsupervised classification applied to overlap derived features provided rates equal to or higher than supervised rates which suggests that knowledge of clinical status may not be necessary for good classification when a suitable selection of structures is used. We identified structures that best separate the groups based on the results of supervised t-tests. Ideally, the selection of structures for analysis should be based on a-priori clinical information on disease-related structures. The selection used in this work has, however, previously been shown to relate to dementia. For example, links between hippocampal, ventricular and thalamic volumes and $\mathrm{AD}$ have been reported (See e.g. 4]).

All the steps described in the framework are intended to be generic and can easily be replaced. In computational terms, segmentation is the costliest step but the remaining steps require little computation. Spectral analysis uses matrices whose order is the number of subjects so the approach remains feasible for studies involving hundreds of subjects. The framework is also readily extended to classification tasks involving more than two groups as the nature of the classifier used is generic.

\section{References}

1. Jack Jr., C., et al.: Medial temporal atrophy on MRI in normal aging and very mild Alzheimer's disease. Neurology 49, 786-794 (1997)

2. Wang, L., et al.: Large deformation diffeomorphism and momentum based hippocampal shape discrimination in dementia of the Alzheimer type. IEEE TMI 26(4), 462-470 (2007)

3. Heckemann, R., et al.: Automatic anatomical brain MRI segmentation combining label propagation and decision fusion. NeuroImage 33(1), 115-126 (2006)

4. Fischl, B., et al.: Whole brain segmentation: Automated labeling of neuroanatomical structure in the human brain. Neuron 33(3), 341-355 (2002)

5. Klöppel, S., et al.: Automatic classification of MR scans in alzheimer's disease. Brain 131(3), 681-689 (2008)

6. Fan, Y., et al.: Spatial patterns of brain atrophy in MCI patients, identified via high-dimensional pattern classification, predict subsequent cognitive decline. NeuroImage 39, 1731-1743 (2008)

7. Crum, W., et al.: Generalized overlap measures for evaluation and validation in medical image analysis. IEEE TMI 25(11), 1451-1461 (2006)

8. Aljabar, P., et al.: Classifier selection strategies for label fusion using large atlas databases. In: Ayache, N., Ourselin, S., Maeder, A. (eds.) MICCAI 2007, Part I. LNCS, vol. 4791, pp. 523-531. Springer, Heidelberg (2007)

9. Leemput, K.V., et al.: Automated model-based tissue classification of MR images of the brain. IEEE TMI 18(10), 897-908 (1999)

10. von Luxburg, U.: A tutorial on spectral clustering. Statistics and Computing 17(4), 395-416 (2007)

11. Duda, R., et al. (eds.): Pattern Classification. Wiley, Chichester (2001)

12. Schott, J., et al.: Measuring atrophy in Alzheimer disease: A serial MRI study over 6 and 12 months. Neurology 65(1), 119-124 (2005)

13. Rueckert, D., et al.: Non-rigid registration using free-form deformations: Application to breast MR images. IEEE TMI 18(8), 712-721 (1999) 